\title{
Sexual issues of women with spinal cord injuries
}

\author{
S W Charlifue MA, K A Gerhart MS, R R Menter MD, G G Whiteneck PhD, M Scott \\ Manley EdD
}

Craig Hospital, 3425 South Clarkson, Englewood, Colorado 80110, USA.

The need for research addressing problems unique to women with spinal cord injuries is well documented. Consequently, 231 such women, ages 18 to 45 , were surveyed. Demographic characteristics and data relating to physician usage, female hygiene, pregnancy, contraception and sexuality were collected.

Analysis revealed that $60 \%$ of the respondents had post injury amenorrhea; the average time until menses resumption was 5 months. The group's post injury pregnancy rate was one-third its pre injury rate, but women with incomplete paraplegia had significantly more pregnancies than those with complete quadriplegia.

Of 47 women who did carry babies to delivery, one-half had vaginal deliveries; $49 \%$ used no anesthesia. Problems during pregnancy included autonomic hyperreflexia, decubitus ulcers, urinary tract infections, water retention, bladder and bowel problems, anemia, spotting, fatigue, cardiac irregularity and toxemia. Many of these problems plagued the women during labor and delivery and in the post partum period as well.

Sixty-nine percent of the women were satisfied with their post injury sexual experiences, although self confidence, spasticity, and lack of spontaneity were issues. Although satisfied with care received from physicians, many women were not content with the information provided during rehabilitation, and felt a need for more literature, counselling, and peer support.

Key words: spinal cord injuries; women; sexual aspects; pregnancy outcome; menstruation.

\section{Introduction}

Turk and Turk write that 'modesty, discretion, and uneasiness' make it difficult to talk about the sexual concerns of women who have spinal cord injuries. ${ }^{1}$ Others have also commented on the limited understanding and unavailability of information pertaining to the sexuality of women with spinal cord injuries. ${ }^{2-8}$

Several explanations may account for the dearth of information available. First, it has been suggested that parts of society may still prefer to see women as weaker and more passive, and disability supports this image. ${ }^{9}$ Also, though less prevalent today, expectations that people with disabilities either subjugate their sexual selves, ${ }^{10}$ or become totally asexual ${ }^{11}$ still persist.

Additionally, among those with spinal cord injuries in particular, women represent a clear minority. ${ }^{12,13}$ Thus, when hospitalized, they are invariably surrounded by male patients, and so may hesitate to ask for and obtain the specialized information they need. ${ }^{13}$ At the same time, many believe that the objective and quantifiable losses of erection, ejaculation, and fertility that males experience merit more attention. ${ }^{14}$ Because a woman's ability to become pregnant and carry and deliver a child is largely unaffected following spinal cord injury, it was assumed that her sexuality was similarly unaffected. ${ }^{15}$ Consequently, female sexuality was equated with child bearing and scientific interest was confined to pregnancy and its complications. ${ }^{1,6}$

Early literature reveals that many patients considered pregnancy following spinal cord injury to be contraindicated, $9,16-18$ with some recommending abortion. ${ }^{18}$ However, 
it is now known that after temporary amenorrhea, fertility returns to normal, $1,1,15,19-21$ and conception, pregnancy and normal labor and delivery are possible, virtually routine, and pose little or no added risk. .,22 $^{1,2}$

Some of the reported complications of pregnancy are severe autonomic hyperreflexia in women with lesions at or above the T6 level, urinary tract infections, anemia, respiratory compromise, and skin breakdown. 1,6,7,9,14,17,18,21,23-27 There may also be an increased risk of early and unattended delivery. ${ }^{25,28}$ Because abdominal muscle paralysis prevents final expulsion of the fetus, there is a relatively high number of forceps-assisted deliveries, and factors like uncontrollable autonomic hyperreflexia, ruptured membranes, and fear of bladder damage contribute to a seemingly higher incidence of Caesarian deliveries. ${ }^{23,26,28}$ Although the risk of miscarriage or birth defects is not increased, there may be a higher than expected rate of still births to women with spinal cord injuries, and average birth weights of their infants may be lower than the general population's average of 3400 grams. ${ }^{1,17,23,26,28}$

Literature addressing other sexuality issues, such as body image, orgasm, desirability, sexual function, and self esteem is much more limited. ${ }^{2,7,8,9}$ However, because spinal cord injury impairs sensation and movement, and alters muscle tone and bladder function, it impacts far more than child bearing. ${ }^{4}$ There are few reports on the effects of these impairments on sexual activity. While some report that women with complete injuries do not have orgasms, ${ }^{18}$ others describe fantasy orgasms, and orgasms emanating from other, unimpaired erogenous zones. ${ }^{19}$ Literature describing the effects of spinal cord injury's impairments on other components of sexuality, such as satisfaction, self image and self esteem are equally rare. Some report that women with a poor pre morbid self image have more trouble adjusting sexually. ${ }^{29,30}$ Others indicate that the complaints of women with spinal cord injuries-not being attractive, not having available partners, and issues relating to comfort, confidence, and relationships - are the same as those of non disabled women. ${ }^{9,29,30}$

Women with spinal cord injuries view sexuality counselling as important to the adjustment process; those with the most complete and accurate information may have fewer emotional problems relating to sexuality. ${ }^{2,11}$ However, once back in the community women complained that if their fertility wasn't compromised, they were made to feel as if nothing else should matter. ${ }^{9}$ They complained that they knew more than or weren't listened to by their physicians, or that they were over-treated. ${ }^{26}$ Physically inaccessible gynecological services were also an issue. ${ }^{16}$

Clearly, women's sexuality is multifaceted, involving relationships, sharing feelings, attractiveness, self confidence, and self worth, as well as pregnancy and child rearing. ${ }^{10}$ Because the current knowledge base of sexuality issues of women with spinal cord injuries is limited, this study was undertaken to examine a wider range of variables.

\section{Methods}

Two hundred and ninety-three women meeting the following study criteria were identified: (1) spinal cord injury, at any neurological level; (2) initial rehabilitation at Craig Hospital, the Rocky Mountain Regional Spinal Injury System, Englewood, Colorado; (3) injury date between January 1, 1959 and December 31, 1983; (4) 18 to 45 years of age in 1984 .

Women were contacted by telephone and participated in a comprehensive survey that examined demographic characteristics, menstrual and female hygiene history, pregnancy and child bearing and sexuality. Two hundred and thirty-one women, or $79 \%$ of the available population, were reached and completed the interview. Data were analyzed descriptively. Tests for statistical significance included the student's t-test and the Chi-square.

\section{Findings}

\section{Demographics}

The mean age of the women at the time of injury was 25.1 years, while at the time of 
the study it was 32.7 years. One hundred and twelve women had quadriplegia and 119 had paraplegia, of whom $72 \%$ and $77 \%$, respectively, had neurologically complete lesions. Wheelchairs were used by $84 \%$, while $16 \%$ walked with or without devices and orthoses.

Seven percent of the women had less than a high school education, $30 \%$ had completed high school, $33 \%$ had attended some college, and $30 \%$ were college graduates or had postgraduate education. In terms of marital status, the number of both marriages and divorces increased after injury (Table I). However, for those married at the time of the study, the mean length of the relationship was 9.8 years.

Thirty-six percent of the women were employed outside the home. Women with incomplete paraplegia had the highest employment rate at $59 \%$, followed by $44 \%$ of those with complete paraplegia and $42 \%$ of those with incomplete quadriplegia. Only $18 \%$ of women with complete quadriplegia were employed. These employment differences based on neurological level were significant $(p<.001)$.

\section{Menstrual and female hygiene history}

Thirteen of the study respondents had undergone hysterectomies prior to their spinal cord injuries. Of the remaining 218, $40 \%$ reported no interruption of their menses following spinal cord injury. For the $60 \%$ reporting amenorrhea, the median time until return of normal menses was just over 5 months (Fig 1). By 12 months $89 \%$ of the women were menstruating, and at 3 years post injury, only one woman had not resumed menses.

Table I Marital statuts

\begin{tabular}{lrc}
\hline Marital status & $\begin{array}{c}\text { Pre-injury } \\
(\%)\end{array}$ & $\begin{array}{c}\text { At time of } \\
\text { study (\%) }\end{array}$ \\
\hline Single & 43 & 29 \\
Married & 37 & 37 \\
Living with partner & 7 & 8 \\
Separated/divorced & 12 & 22 \\
Widowed & 1 & 4 \\
Total & 100 & 100 \\
\hline
\end{tabular}

The majority of women reported little or no change in menstrual regularity, duration, and intensity before and after injury. However, $22 \%$ reported heavier periods, $13 \%$ said periods were longer, and $12 \%$ said they were less regular.

Post injury, $75 \%$ of the women used tampons for menstrual flow control. Fortysix percent reported using external pads either alone or in addition to tampons. Just over $19 \%$ of the women reported problems, including discomfort due to positioning of the tampon or pad, inability to determine if placement was correct and adequate to absorb menstrual flow, and increasing difficulties with catheters and urinary management. Of those who regularly used attendant care, $31 \%$ of the women with quadriplegia and $5 \%$ of those with paraplegia needed more help during their periods.

Forty-three percent of the women reported bowel and bladder changes associated with menstrual periods. These included diarrhea, constipation, increased urine output, water retention, bladder incontinence, and urinary tract infections. Women with indwelling urethral catheters had the fewest bladder changes, while those using intermittent catheterization had the most.

Changes in the amount of vaginal discharge post injury were reported by $53 \%$ of the women. Over $80 \%$ said that discharge

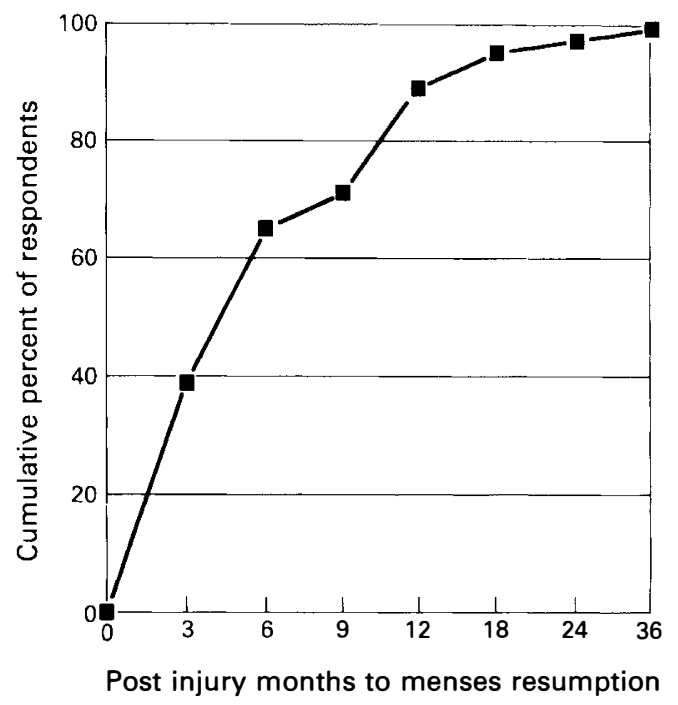

Figure 1 Menses resumption. 
was more profuse, and $76 \%$ reported greater frequency. Treatments, including creams, suppositories and oral medications, were used by $63 \%$. For 9 women no form of treatment could control the problem.

Additionally, $27 \%$ of all the women surveyed reported increased perspiration in the genital area. Many reported using products they hadn't used prior to their injuries, including douches, minipads, powders, sprays and diapers to control vaginal discharge, wetness, odor or discomfort.

\section{Contraception}

At the time of the study, $21 \%$ of the women reported using contraception. The IUD was used most frequently, followed by condoms and birth control pills. Forty-six percent reported problems, including weight gain, headaches and nausea with oral contraceptives and cramping with the IUD.

Of those not using contraception, $44 \%$ indicated they were not sexually active, $5 \%$ were trying to become pregnant, and $6 \%$ cited personal preference. Five percent of the women stated they did not use birth control because they believed they could not get pregnant, or because they were uninformed about contraception. One woman reported having difficulty using birth control. Finally, $38 \%$ of those not using contraception had had pre or post injury tubal ligations or hysterectomies.

Of this latter group, 30 women had undergone the procedure since their injury, half of whom had chosen it as a means of birth control. For 12 women gynecological problems, including benign or malignant tumors, dysmenorrhea, and uterine infections, were the primary indication. One woman cited convenience as the reason, another said it was done in conjunction with bladder removal, and one woman had the procedure on the advice of her physician who feared she would be raped.

\section{Pregancy and child bearing}

Seventy-eight pregnancies were reported, including 6 in women who were pregnant when injured. Three of these 6 carried and delivered their babies; one miscarried at the time of the injury and two had elective abortions. In total, the 78 pregnancies resulted in 47 live births (including 2 sets of twins), 16 miscarriages, and 17 abortions.

The overall post injury pregnancy rate was .34 pregnancies per person, compared with a pre injury rate of 1.30 per person. This decrease is explained, in part, by the fact that over one-third of the women said they did not want children. Of these, $80 \%$ blamed the injury, citing the difficulty they perceived in caring for children from the wheelchair. Also, statistically significant differences were found in pregnancy rates by neurological grouping (Table II). The lower rate for women with complete quadriplegia was consistent with their fewer reported attempts to become pregnant and their decreased rate of sexual activity.

Of those with successful pregnancies, 3 women reported problems with placenta previa, tachycardia and blood type incompatibility in the baby. Problems that the women themselves experienced included decubitus ulcers, urinary tract infections, water retention, bladder and bowel problems, anemia, spotting, fatigue, cardiac irregularity and toxemia. Hyperreflexia was a problem for 9 women; these same 9 patients also had the same condition prior to their pregnancies. For 2 the hyperreflexia episodes were severe.

Fifty-seven percent of the women who carried babies to delivery reported increasing mobility problems. Most often mentioned were losses of independence in activities of daily living (18 women) and increased fatigue ( 5 women). Other problems included increased spasms, inability to drive, lower extremity edema, and pain.

Regarding the labor and delivery period, $77 \%$ of the women who had live births reported being aware of the onset of labor.

Table II Post injury pregnancy rates

\begin{tabular}{lc}
\hline Injury level & Pregnancies/person* \\
\hline Complete quadriplegic & .15 \\
Incomplete quadriplegic & .42 \\
Complete paraplegic & .40 \\
Incomplete paraplegic & .63
\end{tabular}

${ }^{*} p<.001$ 
Labor began prematurely in $19 \%$ of the patients. Eleven women stated that labor was induced, but for 6 this was due to physician preference rather than being a specific medical indication. Types of anesthesia used during delivery included general $(32 \%)$, local $(6 \%)$, spinal $(4 \%)$, epidural block $(2 \%)$, and other forms $(6 \%)$. Fortynine percent of the women did not have an anesthetic.

Fifty-three percent of the women had vaginal deliveries without forceps, while $22 \%$ required forceps assistance. The remaining births were Caesarian deliveries. Five were done by physician choice, 2 were done to deliver transverse-lying twins, 2 more were done due to autonomic hyperreflexia, one was done because of placenta previa, and one because of prolonged labor.

Eleven women had problems with autonomic hyperreflexia during delivery; 7 were treated with medications, including NitroBid, Atropine or Demerol, while 4 were not treated. Other problems reported during labor and delivery included bowel and bladder difficulties, dehydration, and spasticity. In one case, the woman's labor was not taken seriously. Her physician told her that she would be unaware of when labor began; the woman, in fact, was aware, but the physician refused to believe her.

The average birth weight of the infants of these spinal cord injured women was 2980 grams; the smallest baby was 1400 grams, and the largest was 3900 grams. Five of the babies had problems during delivery, including cyanosis, prolapsed cord, heart failure, placenta previa and tachycardia. In the postpartum period 5 babies had jaundice, 2 were born valium addicted, two required incubation, one had an elevated temperature, and one was allergic to the mother's milk. During the first 2 years, one infant died of crib death and another baby, born with severe neurological damage, died of respiratory complications.

Twenty-nine women breastfed their infants. Of the 6 who had difficulties, 4 had insufficient milk, one said her baby was allergic to her milk, and one woman had problems with clogged ducts. Of the 18 women who did not breast feed, most did not do so because of the inconvenience.
However, in 4 cases, the infant was too ill to be breastfed, and in two cases the mother was too ill. Three women reported that their physicians advised them against breast feeding.

Postpartum health or physical problems were reported by just over $10 \%$ of the women, and included bowel and bladder difficulties, spasticity, thrombophlebitis, fatigue, anemia, and headaches. Also, over half of the women reported that they needed more physical assistance in the home.

\section{Sexual activity}

Prior to injury $76 \%$ of the women reported having sexual contact at least once a week. After injury this decreased to $52 \% ; 34 \%$ had sexual contact less than once a month. Although women with complete quadriplegia had the fewest sexual contacts, the differences between the 4 neurological groups were not significant.

The women reported participating in various sexual activities since injury, the most frequent being genital intercourse $(77 \%)$, oral-genital intercourse $(63 \%)$, and fantasy $(45 \%)$. Overall, $69 \%$ were satisfied with their post injury sexual experiences.

Approximately half of the women reported that they had experienced orgasms since their injuries. The stimulus was generally genital or a combination of genital and breast stimulation, although 13 women reported orgasm resulted from stimulation above the level of injury. More than half of the women indicated that the methods of stimulation used were the same before injury, but $43 \%$ reported that more time was required to achieve orgasm. Forty-three percent said the intensity of orgasm was less while $42 \%$ felt frequency was decreased. There was a statistically significant difference between those with paraplegia and those with quadriplegia $(p<.05)$, with $60 \%$ of the former reporting orgasms compared with $40 \%$ of the women with quadriplegia.

Spasticity was identified as a factor that interfered with sexual activity by $21 \%$ and the lack of spontaneity interfered for $17 \%$. Twelve percent cited range of motion limits, $10 \%$ listed catheter interference, $9 \%$ reported inadequate vaginal lubrication, and 
$7 \%$ reported that autonomic hyperreflexia were interfering factors. The bowel program, medications, and the presence of the attendant were also factors for a small number.

Although most women felt they and their partners had adjusted well to disability related sexuality issues, 26 reported that their partners were either unwilling to discuss concerns, or were unwilling to continue sexual contact. For $46 \%$ of the women their own priorities had changed, causing sex to be less important to them than it had been prior to the injury.

Twenty-five percent of the women felt self confidence played a role in their sexual activity and adjustment. Self image was also a factor, and was assessed using the bipolar adjectives: attractive/unattractive, desirable/undesirable, and sexually skilled/sexually unskilled. Respondents were asked to rate themselves on $0-10$ scales both pre and post injury. Table III depicts the differences in their pre and post injury ratings.

Overall, perceptions of desirability and attractiveness were lower post injury, even though $35 \%$ of the respondents indicated that they felt better about themselves as women than they did prior to their injuries. Thirty-eight percent felt worse about themselves, and $27 \%$ said they felt the same. Women with quadriplegia rated themselves lower in sexual skill than prior to injury, while those with paraplegia rated themselves slightly higher. Further analysis revealed that women with positive attitudes about themselves had also conceived and delivered more children post injury, reported more frequent sexual activity, and had fewer problems with hygiene.

\section{Sexuality information and follow up}

More than half of the women reported that the sexuality information provided for them during rehabilitation was inadequate. However, those whose rehabilitation had been after 1977 had higher levels of satisfaction. This coincided with the establishment of a weekly women's group at the spinal injury treatment center.

After discharge from rehabilitation, $75 \%$ of the women reported routine physician contact for female health care needs. Only $7 \%$ of the women listed financial or insurance problems as the reason for their failure to maintain routine gynecological follow up care. Relationships with physicians generally were satisfactory. Fifty-five percent of the women said their local physicians had a good understanding of their needs; $64 \%$ felt their physicians were comfortable working with them. Of those women who reported that their physicians were not comfortable with them, most cited a 'general lack of spinal cord injury knowledge' as the reason. Physician office inaccessibility was an issue, with over $40 \%$ of the women with quadriplegia and neurologically complete paraplegia reporting difficulty getting on and off examination tables.

The women also reported that they were comfortable discussing sexuality issues with others. Although $22 \%$ had sought individual and/or group sexuality counselling, all reported that they could discuss sexuality issues with family, friends, and/or other women with spinal cord injuries. However, $11 \%$ asked for more information from other women with spinal cord injuries, and $24 \%$ indicated that additional literature and films would be a helpful source of information.

\section{Discussion}

There clearly is a perceived need for more and better information and counselling services for women with spinal cord injuries, despite the fact that there may have been improvements in recent years. This study,

Table III Self image

\begin{tabular}{lccc}
\hline & $\begin{array}{c}\text { Mean value } \\
\text { pre injury }\end{array}$ & $\begin{array}{c}\text { Mean value } \\
\text { post injury significance }\end{array}$ \\
\hline Attractiveness & 7.76 & 6.94 & $p<.001$ \\
Desirability & 7.76 & 6.59 & $p<.001$ \\
Sexual skill & 7.02 & 6.57 & $p<.05$ \\
\hline
\end{tabular}


by documenting self image changes, and issues and concerns regarding sexual activity, contraception, pregnancy, and child rearing, may be of help in identifying specific areas where professional and peer support can be of benefit.

However, counselling and information are not enough. Perhaps as important are other issues identified by this study which may require unique rehabilitation interventions. Skin concerns - not only as a result of the changing body composition, fatigue, and increasing immobility of pregnancy, but also from documented post injury increases in vaginal discharges and perspiration-are one example. There needs to be recognition that women's risk factors and needs in this area differ from men's. Education, treatment and even equipment interventions will need to be identified and evaluated to address skin concerns.

Spasticity was also a concern for these women. Though numbers are small, it was a concern during sexual activity, during pregnancy, during delivery, and in the postpartum period. Also problematic is the appearance of valium addiction in 2 of the newborns of these women. Clearly, the management of spasticity for spinal cord injured women in their child bearing years merits continued attention.

In addition to antispasmodics, other medications routinely used by persons with spinal cord injuries require further study. The effects of all these agents on the pregnant woman, her fetus, and on the infant who is breast fed were not addressed in this and other studies, but do need much closer scrutiny.

Contraceptive options for women with spinal cord injuries also need closer examination. The use of oral contraceptives may be contraindicated because of the already higher risk of clots and deep venous thromboses. Similarly, sensory impairment may make the use of intrauterine devices dangerous, and for those women with limited hand function, independent placement of the diaphragm may not be feasible. ${ }^{2,9}$ In addition, the possibility that hysterectomy and tubal ligation are used, in at least some cases, out of ignorance, also needs attention.
Finally, increased dependence and the need for additional physical assistance during pregnancy, as well as during the postpartum period, are needs that have not been addressed, but for which action is needed. Even with additional help, caring for newborn and older children is difficult and indeed overwhelming for many women who use wheelchairs. That this is a far reaching concern is made clear both by the reports of new mothers, and by reports that other women are not even considering pregnancy because of perceived child rearing difficulties. The importance of motherhood to a disabled woman's quality of life needs to be acknowledged, and additional physical assistance may need to be offered to make this goal attainable.

The limitations of this study are those inherent to a survey methodology. Although the instrument was comprehensive, it did not allow for follow up or elaboration. Future studies might limit the ages and times post injury of respondents, and the time period encompassed by the survey. This particular study included women who were injured many years ago and so made contraceptive and other decisions in an era of considerably less medical enlightenment, as well as very young women, recently injured, who had not yet faced many of the issues raised by the survey. In addition, comparative administration of the survey to a non disabled control group might also be considered.

Nonetheless, these findings are useful in identifying directions for research. Because of the relatively small numbers of women with spinal cord injuries-and the even smaller numbers experiencing the problems described in this report-multicenter collaborative research may be needed. Controlled studies using specific treatment approaches and interventions might be conducted. The women themselves, along with their rehabilitators, will then need to assume leadership roles to insure that findings are disseminated so that their needs and concerns are addressed.

\section{Acknowledgements}

This research was supported by a United States 
government National Institute on Disability and Rehabilitation Research grant, No. G008535132. The authors wish to thank the many women who took considerable time to contribute to this research. Appreciation is extended to Pris- cilla Henry RN for her extensive assistance in collecting the data for this study. Thanks are also extended to Susan Solnick and George Richardson for their assistance with data collection.

\section{References}

1 Turk R, Turk M, Assejev V (1983) The female paraplegic and mother-child relations. Paraplegia 21: 186-191.

2 Becker EF (1979) Sexuality and the spinal-cord-injured woman: an interview. Sex Disabil 2(4): 278-286.

3 Berard EJJ (1989) The sexuality of spinal cord injured women: physiology and pathophysiology: a review. Paraplegia 27: 99-112.

4 Francois N, Maury, M (1987) Sexual aspects in paraplegic patients. Paraplegia 25: 289-292.

5 Griffith ER, Tomko MA, Timms RJ (1973) Sexual function in spinal cord-injured patients: a review. Arch Phys Med Rehabil 54: 539-543.

6 Griffith ER, Trieschmann, RB (1975) Sexual functioning in women with spinal cord injury. Arch Phys Med Rehabil 56: 18-21.

7 Letcher JC, Goldfine LJ (1986) Management of a pregnant paraplegic in a rehabilitation center. Arch Phys Med Rehabil 67: 477-478.

8 Thornton CE (1979) Sexuality counseling of women with spinal cord injuries. Sex Disabil 2(1): $267-277$.

9 Cole TM (1975) Sexuality and physical disabilities. Arch Sex Behav 4(4): 389-403.

10 Romano MD (1978) Sexuality and the disabled female. Sex Disabil 1(1): 27-33.

11 Ray C, West B (1984) Social, sexual and personal implications of paraplegia. Paraplegia 22: 75-86.

12 Kennedy EJ (1986) Spinal Cord Injury: the Facts and Figures. University of Alabama at Birmingham, Birmingham, AB: 13.

13 Scheele C (1988) Women \& SCI: part 1: overview. Paraplegia News: 41-43.

14 Johnston B (1982) Pregnancy and childbirth in women with spinal cord injuries: a review of the literature. Matern Child Nurs J 11(1): 41-45.

15 Axel SJ (1982) Spinal cord injured women's concerns: menstruation and pregnancy. Rehabil Nurs 7(5): $10-15$.

16 Asrael W (1982) An approach to motherhood for disabled women. Rehabil Lit 43(7-8): 214-218.

17 McGregor JA, Meeuwsen J (1985). Autonomic hyperreflexia: a mortal danger for spinal cord-damaged women in labor. Am J Obstet Gynecol 151(3): 330-333.

18 Ohry A, Peleg D, Goldman J, David A, Rozin R (1978) Sexual function, pregnancy and delivery in spinal cord injured women. Gynecol Obstet Invest 9: 281-291.

19 Comarr AE (1985) Sexuality and fertility among spinal cord and/or cauda equina injuries. J Am Paraplegia Soc 8(4): 67-75.

20 Durkan JP (1968) Menstruation after high spinal cord transection. Gynecology 100(4): 521-524.

21 Silver JR (1975) Sexual problems in disorders of the nervous system. Br Med J 3: 480-482.

22 Tabash KMA, Brinkman CR, Reff, RA (1892) Autonomic dysreflexia in pregnancy. Obstet Gynecol 60(1): 119-121.

23 Bluestein D, Starling E, Moore P, Droesch J, Davis, D, Wade W (1988) Caring for a paraplegic patient and her family. J Fam Pract 27(4): 365-371.

24 Greenspoon JS, Paul RH (1986) Paraplegia and quadriplegia: special considerations during pregnancy and labor and delivery. Am J Obstet Gynecol 155(4): 738-741.

25 Wanner MB, Rageth CJ, Zach GA (1987) Pregnancy and autonomic hyperreflexia in patients with spinal cord lesions. Paraplegia 25: 482-490.

26 Verduyn, WH (1986) Spinal cord injured women, pregnancy and delivery. Paraplegia 24: 231-240.

27 Rudolph AM (1982) Pediatrics, 17th ed. Appleton-Century-Croft, New York.

28 Robertson DNS (1972) Pregnancy and labor in the paraplegic. Paraplegia 16(3): 209-212.

29 Bregman S (1978) Sexual adjustment of spinal cord injured women. Sex Disabil 1(2): 85-92.

30 Zwerner J (1982) Yes we have troubles but nobody's listening: sexual issues of women with spinal cord injury. Sex Disabil 5(3): 158-171. 